
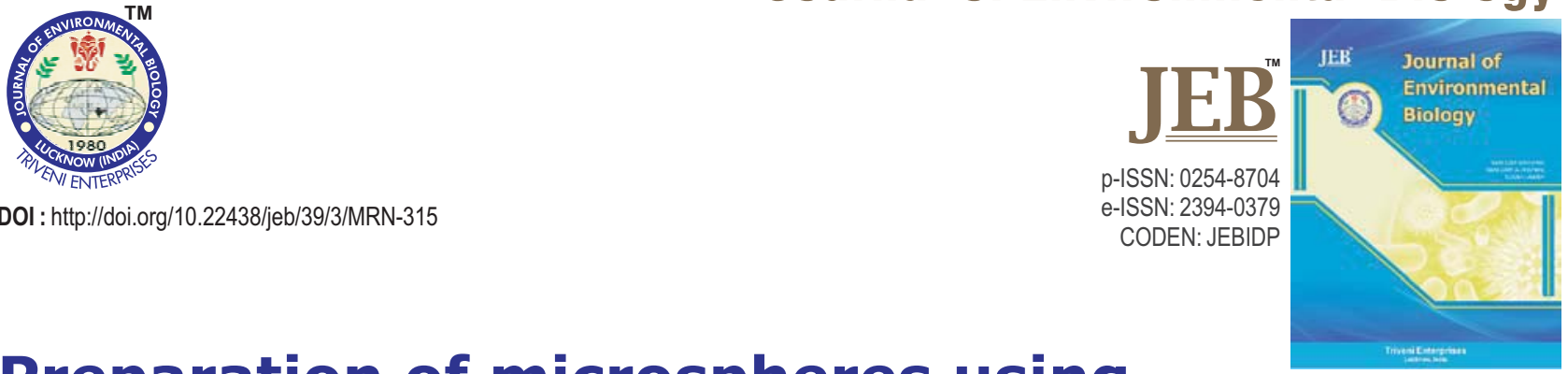

\title{
Preparation of microspheres using poly-3-hydroxybutyrate biopolymer and its characterization
}

\begin{abstract}
Authors Info
C. Swornakumari, S. Meignanalakshmi*, R. Legadevi and A. Palanisammi Department of Animal Biotechnology, Madras Veterinary College, Tamil Nadu Veterinary and Animal Sciences University, Chennai-600 007, India

*Corresponding Author Email : smeignanalakshmi@gmail.com
\end{abstract}

Key words

Controlled drug release

Drug encapsulation

Gentamicin

Poly-3-hydroxybutyrate

\section{Publication Info}

Paper received : 06.09.2016

Revised received : 25.11 .2016

Re-revised received: 04.08 .2017

Accepted : 10.10 .2017

\begin{abstract}
Aim : Biodegradable poly-3-hydroxybutyrate is one of the most common biopolymer which is used in
\end{abstract} different fields such as medicine, agriculture, textile, industrial and food packaging. PHB microspheres are useful for targeting drugs to specific infection sites and for prolonged drug release. The present study focus on microsphere preparation for effective controlled drug release using poly-3hydroxy butyrate biopolymer.

Methodology : In the present study, poly3-hydroxy butyrate microspheres were prepared using solvent evaporation technique and characterized by SEM and FTIR. Poly-3-hydroxy butyrate microspheres were encapsulated with BSA and gentamicin. Drug encapsulation efficiency was determined. In vitro drug release profile and in vitro cytotoxicity was also studied.

Results : The prepared microspheres were of $2 \mu \mathrm{m}$ in size. Microspheres fabricated with $1 \%$ polyvinylalcohol showed encapsulation efficiency of $94.3 \%$ and $90.27 \%$ with BSA and gentamicin, respectively. The in vitro release studies in simulated body fluid, phosphate buffered saline and contact lens solution showed initial burst release followed by controlled release. In vitro cytotoxicity analysis showed $98 \%$ and $95 \%$ viability of cells in 3T3L1 cell line for microsphere encapsulated with BSA and gentamicin, respectively.

Interpretation : Poly-3-hydroxy butyrate microspheres were found to release BSA and gentamicin in a controlled manner Preparation of microsphere using poly-3-hydroxy butyrate by solvent evaporation technique (PHB)

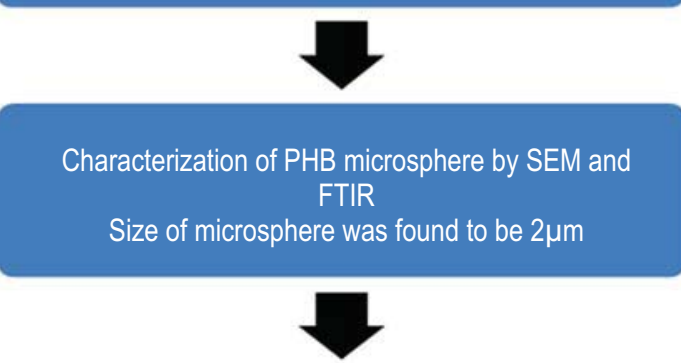

Encapsulation of PHB microsphere with BSA and Gentamicin and the encapsulation efficiency was $94.3 \%$ and $90.27 \%$ respectively

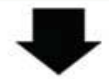

Studies on in vitro drug release from PHB microspheres revealed initial burst release followed by controlled drug release

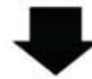

PHB microspheres were found to be non-toxic when tested for in vitro cytotoxicity using 3T3L1 cell line and were found to be non-toxic by in vitro cytotoxicity studies. 


\section{Introduction}

Biopolymers are a type of polymers that are degradable and environmentally safe. Polymers have an undesirable influence on the environment and cause problems with waste deposition and utilization (Emily and Rolf, 2013). There is a need to substitute for polymer having the ability to undergo biodegradation processes. Biodegradable polymers are gaining increasing attention due to their properties such as biocompatibility, nontoxic and high degradability in different environments and processing versatility. Biodegradable poly-3hydroxybutyrate (PHB) is considered as one of the most common intracellular biopolymer first discovered in bacteria (Muralidharan and Radha, 2015) and is used in different fields such as medicine, agriculture, textile, industrial and food packaging (Siracusa et al., 2008).

This biopolymer is used in drug delivery systems in the form of microsphere (Atul, 2014; Aggarwal et al., 2012). PHB microspheres are useful for targeting drugs to specific infection sites and for prolonged drug release (Mao et al., 2012; Joshi and Patel, 2012). The product of degradation of PHB is 3hydroxy butyric acid which is normally present in human blood and this is advantageous for PHB to be used in medical field (Siraj et al., 2014). Controlled drug release which increase the therapeutic activity, decrease the side effects and reduce the number of dosages required during the treatment. Factors such as morphology, size of particles, their chemical composition, rate of degradation and the type of drug affects the drug delivery, by controlling these factors drug delivery can be controlled (Grillo et al., 2011). Several different kinds of drugs can benefit from controlled delivery, such as anti-inflammatory agents, antibiotics, chemotherapeutic drugs, immune suppressants, anesthetics and vaccines. PHBs have been used for a number of biomedical applications such as wound management, orthopaedics, drug delivery and vascular system applications (Giovana et al., 2012). In view of the above, in the present study, microspheres were prepared by using PHB biopolymer and characterized for controlled drug release by encapsulating with BSA and gentamicin.

\section{Materials and Methods}

PHB biopolymer produced by Bacillus sp. available in laboratory was used for the preparation of microspheres.

Microsphere preparation : PHB microspheres were prepared by triple emulsion solvent evaporation technique. $600 \mathrm{mg}$ PHB and $200 \mu \mathrm{l}$ of polyethylene glycol (PEG) were dissolved in $10 \mathrm{ml}$ of dichloromethane. To the mixture, $1 \mathrm{ml}$ of $6 \%$ gelatin solution at $40^{\circ} \mathrm{C}$ was added and the mixture was shaken. Then, $150 \mathrm{ml}$ of $0.5 \%$ or $1 \%$ PVA was added (poly vinyl alcohol) and stirred mechanically with different stirring rate 600,800 and $1000 \mathrm{rpm}$ until the solvent was completely evaporated. After $24 \mathrm{hrs}$ of stirring, microspheres were collected by centrifuging at 5,000 rpm for 15 min and rinsed in distilled water for 7-8 times (Shishatskaya et al., 2008).

\section{Characterization of PHB microspheres}

Scanning Electron Microscope analysis : Surface morphology of PHB microspheres was analyzed using scanning electron microscope (HITACHI model S-3000H).

Fourier transform infrared spectroscopy analysis for PHB and PHB microspheres: PHB polymer and PHB microspheres were subjected to Fourier Transform Infrared Spectroscopy analysis by FTIR spectrophotometer. Spectra were documented in $4000 \mathrm{~cm}^{-1}$ to $400 \mathrm{~cm}^{-1}$ range

Determination of residual PVA content for drug loading efficiency: Two $\mathrm{mg}$ of microspheres were treated with $2 \mathrm{ml}$ of $0.5 \mathrm{M}$ sodium hydroxide for $15 \mathrm{~min}$ at $60^{\circ} \mathrm{C}$. To the mixture, $900 \mu \mathrm{l}$ of $1 \mathrm{~N}$ hydrochloric acid was added to neutralize the samples and the volume was adjusted to $5 \mathrm{ml}$ with distilled water. To the mixture, $3 \mathrm{ml}$ of $0.65 \mathrm{M}$ boric acid, $0.5 \mathrm{ml}$ iodine solution and $1.5 \mathrm{ml}$ of distilled water were added. After 15 min incubation, absorbance was measured at $690 \mathrm{~nm}$.

Encapsulation of PHB microsphere with BSA and gentamicin : Microsphere preparation (1\% PVA at $800 \mathrm{rpm}$ ) procedure was followed with addition of BSA and gentamicin along with PHB (Shishatskaya et al., 2008)

In vitro drug release studies for PHB microsphere encapsulated with BSA and gentamicin : Five mg of BSA and gentamicin loaded microspheres were immersed separately in $3 \mathrm{ml}$ of contact lens solution, phosphate buffer (PBS) (pH, 7.2) and simulated body fluid (SBF) (Kokubo et.al., 1990). The Composition of simulated body fluid consisted pure water, sodium chloride, sodium bicarbonate, potassium chloride, potassium phosphate dibasic trihydrate, magnesium chloride hexahydrate, calcium chloride, Sodium sulphate and Tris base. They were kept in a shaker at $250 \mathrm{rpm}$ at $37^{\circ} \mathrm{C}$. About $1 \mathrm{ml}$ of the sample was collected at regular intervals, till $24 \mathrm{hrs}$. Each sample was replaced with fresh medium and the tubes were returned to the shaker. At each time point, samples were taken out in triplicates and the results were averaged. The BSA and gentamicin content in the aliquot was determined by Coomassie brilliant blue and measuring the absorbance at 450 $595 \mathrm{~nm}$ and quantified using BSA and gentamicin standard curves.

Encapsulation efficiency of BSA and gentamicin : Ten mg of BSA and gentamicin loaded microspheres were dispersed in chloroform with vigorous shaking at room temperature for $24 \mathrm{hrs}$. The samples were collected at $0 \mathrm{hr}$ and 24hr, BSA and gentamicin content were determined by Coomassie brilliant blue by measuring the absorbance at 450-595 $\mathrm{nm}$. Encapsulation efficiency was determined by the following formula: 
Encapsulation efficiency \% $=\frac{\text { Retained BSA/Gentamicin amount }}{\text { Initially loaded BSA/Gentamicin amount }}$

In vitro cytotoxicity study : In vitro cytotoxicity of $\mathrm{PHB}$ microsphere (prepared with 1\% PVA at $800 \mathrm{rpm}$ ), PHB microspheres encapsulated with BSA and gentamicin, was carried out using 3T3L1 cell lines by MTT assay at different concentrations (1 $\mathrm{mg}, 10 \mathrm{mg}$ and $100 \mathrm{mg}$ ). 3T3L1 cell lines were commonly used for in vitro cytotoxicity studies. Briefly, 3T3L1 cell line was subjected to serum starvation for 3-4 hrs followed by incubation with various concentrations ( $1 \mathrm{mg}, 10 \mathrm{mg}$ and $100 \mathrm{mg}$ ) of samples for $24 \mathrm{hrs}$. After $24 \mathrm{hrs}$ of incubation, $100 \mu \mathrm{l}$ of $0.01 \%$ triton X-100 was added to positive control well and incubated for $30 \mathrm{~min}$. The medium from the wells was completely aspirated and $100 \mu$ l of $1 X$ PBS was added to the wells and incubated for $10 \mathrm{~min}$. A $20 \mu \mathrm{l}$ of MTT (3-[(4, 5-dimethylthiazol-2-yl)-2, 5-diphenyl tetrazolium bromide]) was added to the wells and incubated for $4 \mathrm{hrs}$ in dark at $37^{\circ} \mathrm{C}$. A $100 \mu$ l of solubilization buffer (DMSO) was added to the wells and incubated in dark for $1 \mathrm{hr}$. Absorbance was measured at $570 \mathrm{~nm}$ and percentage cell viability was determined.

\section{Results and Discussion}

The SEM analysis of microspheres prepared by using PHB revealed the presence of uniform spherical shaped microspheres of $2 \mu \mathrm{m}$ size (Fig.1). In the present study, dichloromethane was selected instead of chloroform due to lesser miscibility of chloroform in water as compared to dichloromethane, as the type of solvent influences the surface morphology of the microsphere. Siraj et al. (2014) used chloroform as a solvent for PHB microsphere production and obtained a spherical shaped porous microspheres of 10-50 $\mu \mathrm{m}$ in diameter with rough surface. In the present study, use of dichloromethane resulted in uniform spherical shaped microspheres of smaller size when compared to chloroform Shishatskaya et al. (2008) also reported uniform spherical shaped microspheres when dichloromethane was used.

The FTIR analysis showed that, the peak which is assigned for PHB material such as $1647.5 \mathrm{~cm}^{-1}$ and $1238.3 \mathrm{~cm}^{-1}$, $1402.2 \mathrm{~cm}^{-1}, 1238.3 \mathrm{~cm}^{-1}, 1076.7 \mathrm{~cm}^{-1}$ and $3447.50 \mathrm{~cm}^{-1}$ were present in the $\mathrm{PHB}$ microsphere (Fig.2), Functional groups $\mathrm{C}=0$, $\mathrm{CH}_{3}, \mathrm{CH}_{2}$ present in the $\mathrm{PHB}$ microsphere indicates the presence of $\mathrm{PHB}$ and without any disturbances or merging of solvent functional groups in the PHB microsphere.

FTIR spectrum of PHB microsphere encapsulated with gentamicin (Fig.3) showed characteristic peaks of both PHB microsphere and gentamicin, which confirms the presence of gentamicin within the microsphere. FTIR spectrum of PHB microsphere encapsulated with BSA (Fig.4) showed characteristic peaks of both PHB microsphere and BSA, which confirmed the presence ofBSA within the microsphere.

When PVA was used at $0.5 \%$ and $1 \%$ concentration with $800 \mathrm{rpm}$ stirring rate, uniform spherical shaped microspheres were obtained. PVA at $0.5 \%$ and $1 \%$ concentration with stirring rate at $600 \mathrm{rpm}$ and $1000 \mathrm{rpm}$ resulted in microsphere with breakage and also the shape of the microspheres were not spherical and uniform. The residual PVA content of microspheres were higher, when microspheres were prepared at stirring rate $800 \mathrm{rpm}$ when compared to stirring rate at $600 \mathrm{rpm}$ and $1000 \mathrm{rpm}$ (Fig.5). Quantity of PVA influences the microsphere formation because of their hydrophilicity and miscibility in water.

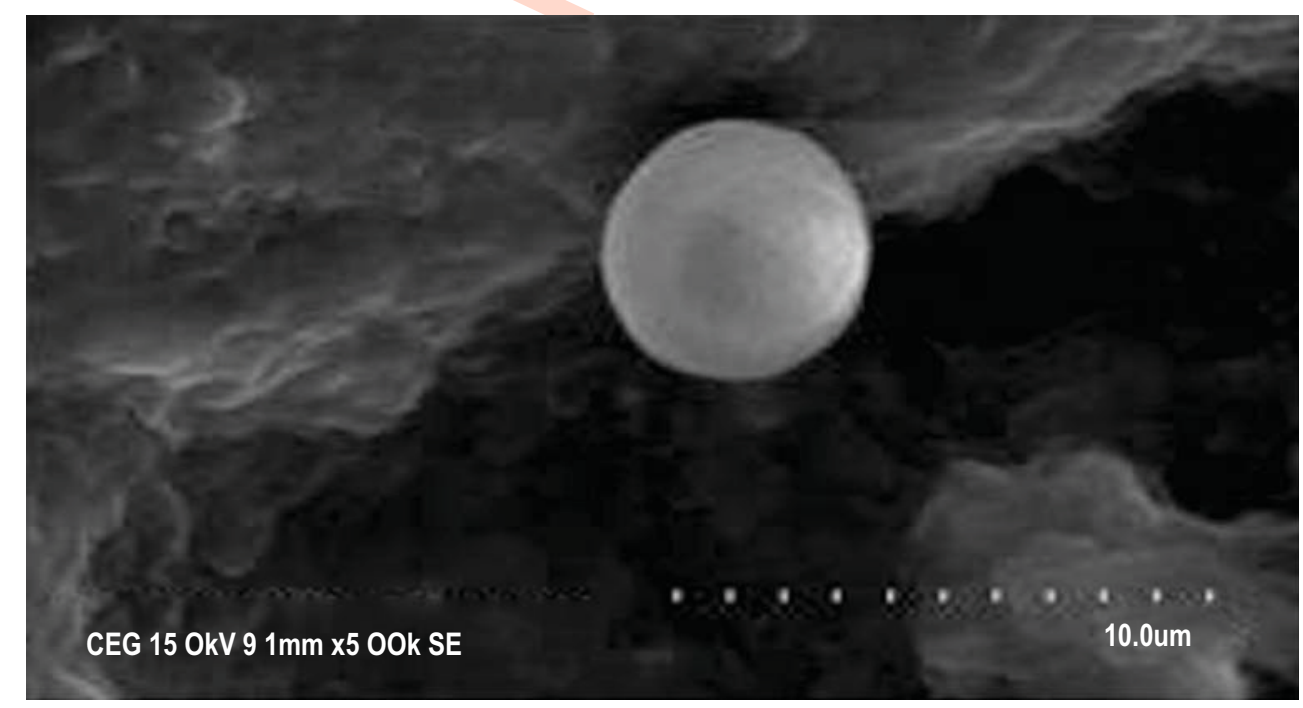

Fig. 1 : Scanning Electron Micrograph of PHB microspheres 


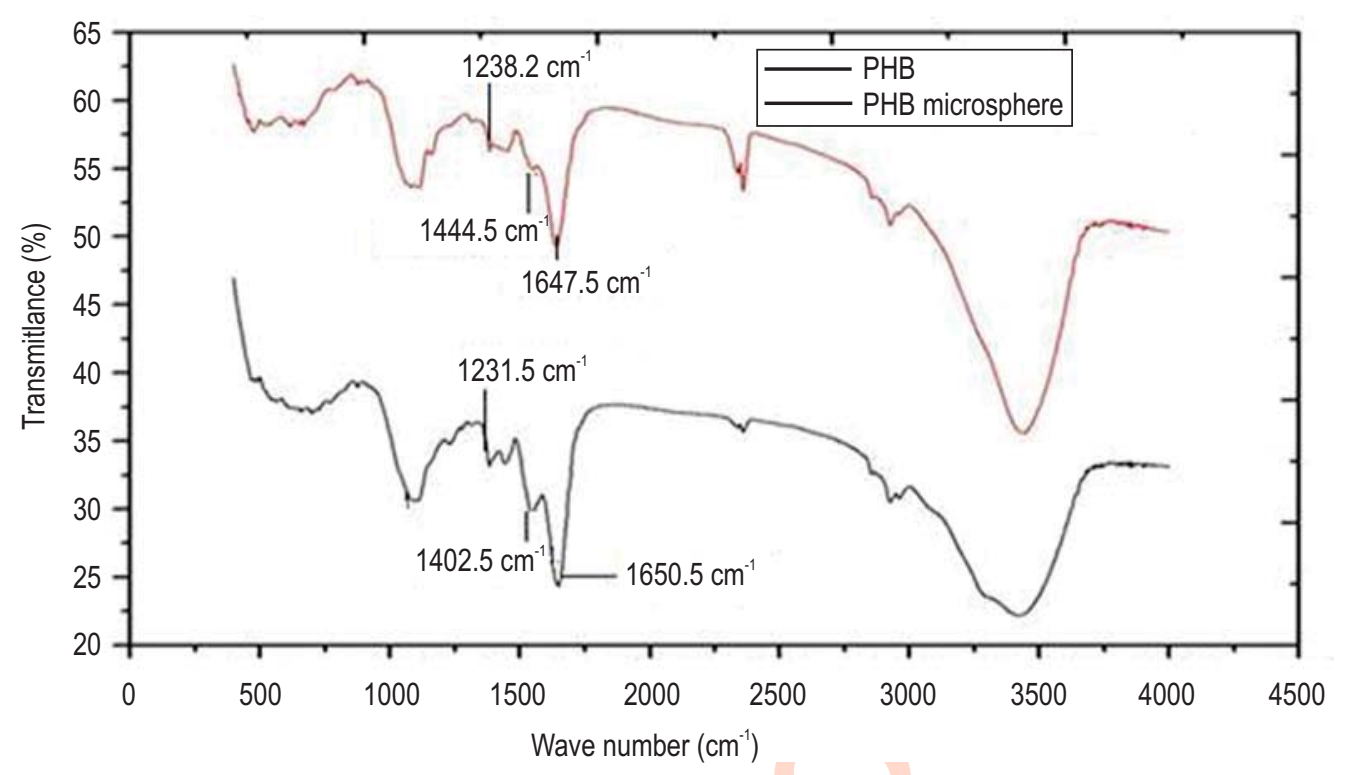

Fig. 2 : FTIR spectrum of PHB and PHB microspheres

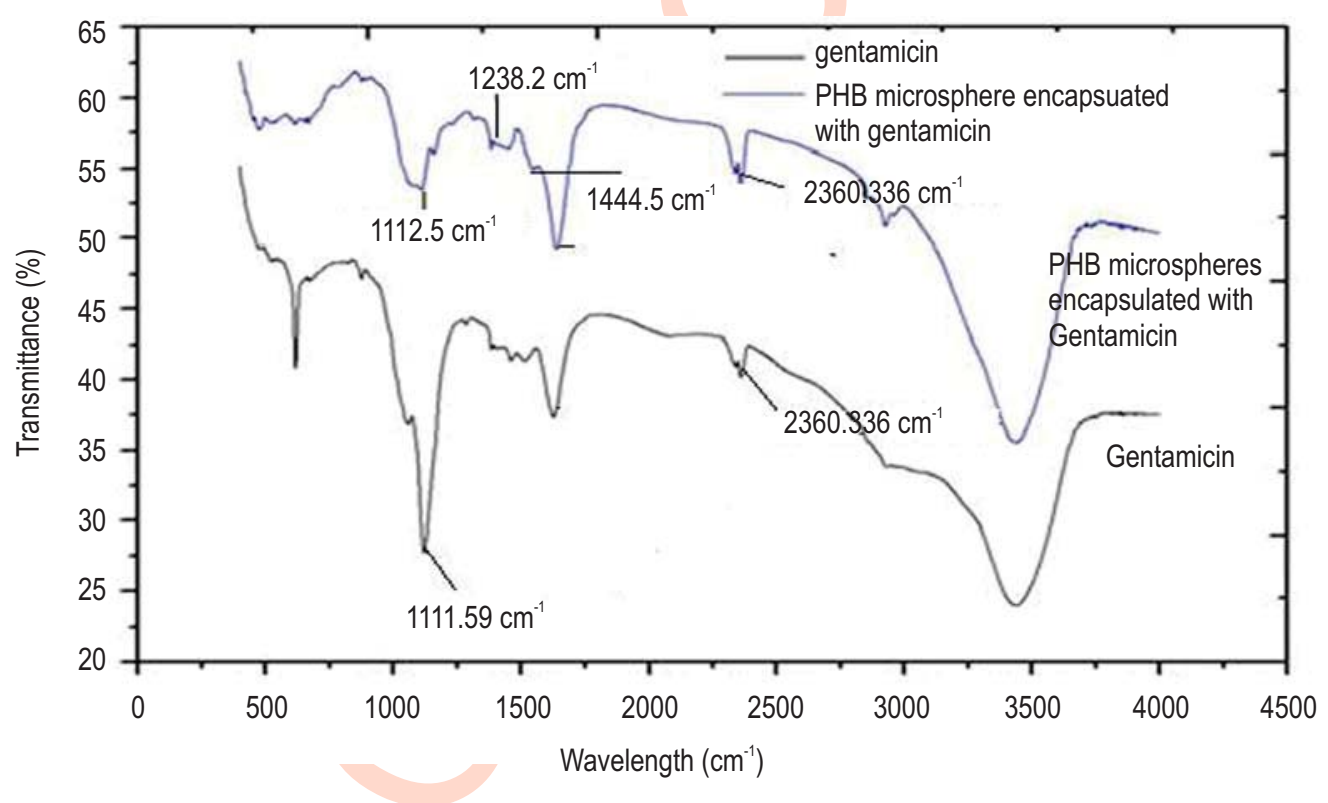

Fig. 3 : FTIR spectrum of gentamicin and PHB microsphere encapsulated with gentamicin

Encapsulation efficiency of BSA and gentamicin (Fig.6) was found to be $94.3 \%$ and $90.27 \%$ when PVA was used at $1 \%$ concentration with a stirring rate of 800rpm. When PVA was used at $1 \%$ concentration with stirring rate $600 \mathrm{rpm}$ and $1000 \mathrm{rpm}$, the encapsulation efficiency of gentamicin was found to be $77.09 \%$ at $600 \mathrm{rpm}$ and $88.64 \%$ at $1000 \mathrm{rpm}$. The encapsulation efficiency of BSA was found to be $89 \%$ at $600 \mathrm{rpm}$ and $82 \%$ at $1000 \mathrm{rpm}$. Similarly, Francis et al. (2011) also observed maximum encapsulation efficiency of drugs of about $48 \%$ using 1\% PVA at 800 rpm. Yang et al. (2001) also reported that 1\% PVA concentration resulted in higher encapsulation of drugs when compared with lower encapsulation with 0.5\% PVA concentration. Hence, the microsphere preparation was proceeded with $1 \%$ PVAconcentration.

The in vitro release of PHB microsphere encapsulated with gentamicin and BSA is shown in Fig. 7 and 8 . In the present study, the initial burst release followed by controlled release was observed with PHB microspheres encapsulated with gentamicin and BSA. Initial burst ( 0 to $1 \mathrm{hr}$ ) release was found to be $30.4 \%$, 


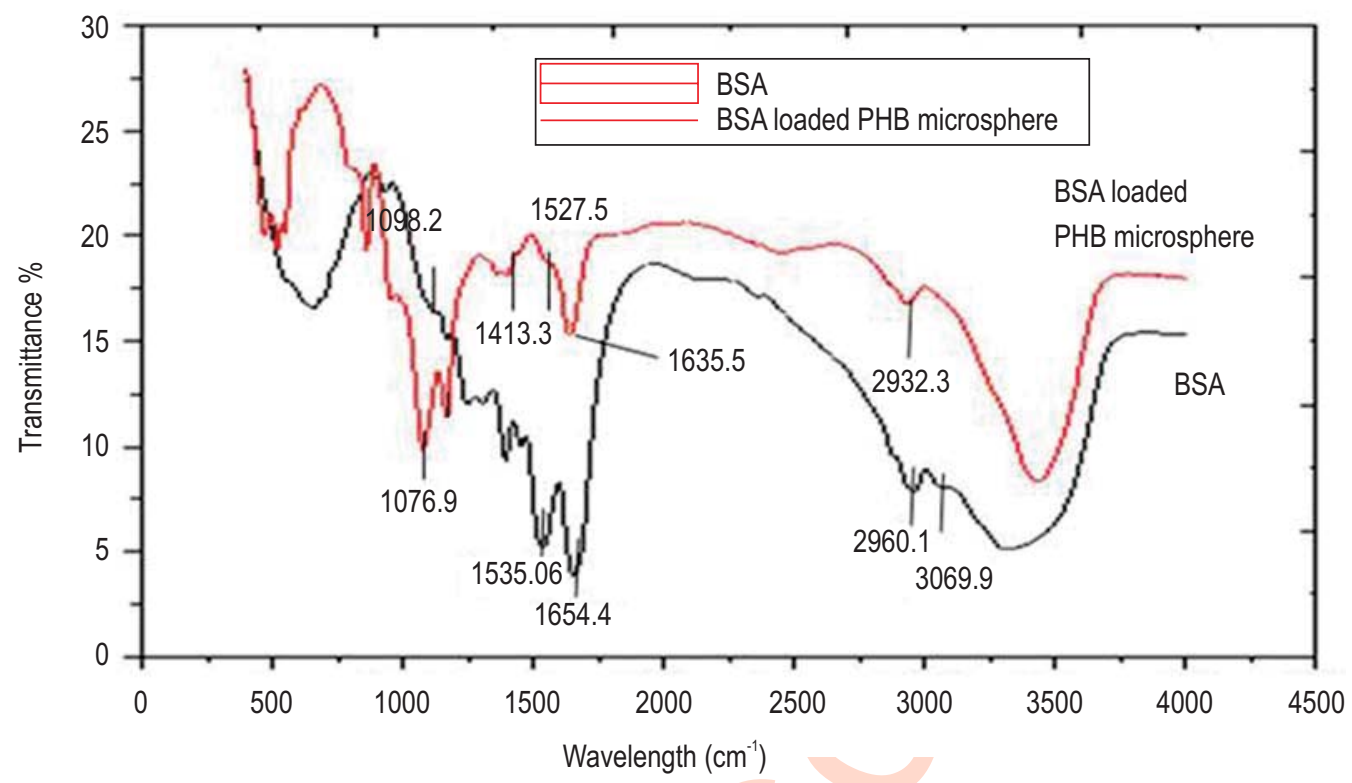

Fig. 4 : FTIR spectrum of BSA and BSA loaded PHB microsphere

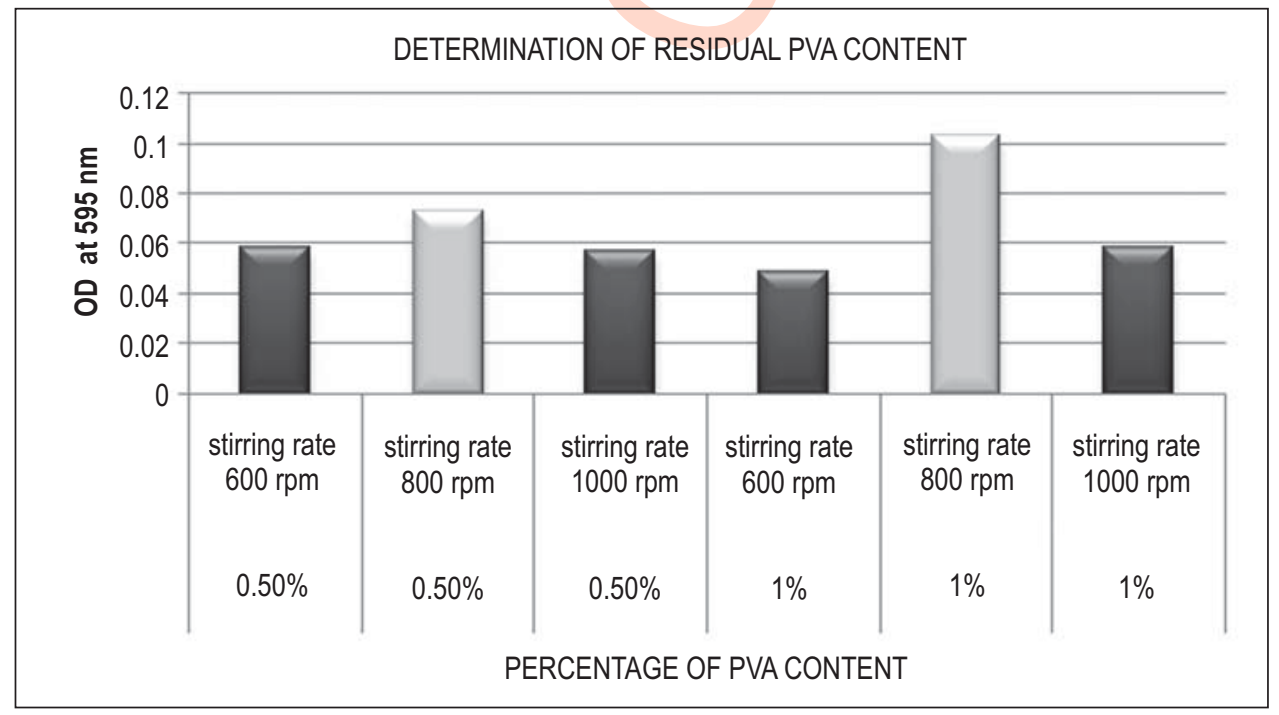

Fig. 5 : Residual PVA content at different concentration of PVA at different stirring rate during microsphere preparation

$31.89 \%, 32.915 \%$ in SBF, PBS and CLS for BSA and $30 \%, 31 \%$, $29.7 \%$ in SBF, PBS and CLS for gentamicin. This release continued up to $24 \mathrm{hr}$ and the total release was found to be $78.86 \%, 75.24 \%$ and $72.91 \%$ in SBF, PBS and CLS for BSA. The total release for gentamicin was found to be $79 \%, 77.4 \%$ and $80.8 \%$ in SBF, PBS and CLS, respectively. Similarly, Francis et al. (2011) observed the in vitro drug release of gentamicin in simulated body fluid at $37^{\circ} \mathrm{C}$ for a period of $20 \mathrm{hr}$. An initial burst release of $90.6 \mu^{-1} \mathrm{gml}^{-1}$ at $0-1 \mathrm{hr}$ was reported, which was $60 \%$ of the total encapsulated drug followed by the controlled drug release upto $20 \mathrm{hrs}$. Controlled drug release was found to be $24.6 \mu \mathrm{gml}^{-1}$ at $5 \mathrm{hr}$ and $18 \mu \mathrm{gml}^{-1}$ at $12 \mathrm{hrs}$. After $20 \mathrm{hrs}$, the total drug release of $95.33 \%$ was noted.

Ismail et al. (2012) observed the in vitro release of gentamicin in PBS at $37^{\circ} \mathrm{C}$ for a period of 6 days at a regular interval of $1 \mathrm{hr}$. Approximately, $50 \%$ of gentamicin was released within $10 \mathrm{hrs}$ and release continued to be minimal for next few hrs with total release of less than $60 \%$ for a period of 6 days. Rossi et al. (2004) observed the release of gentamicin in phosphate buffered saline at $37^{\circ} \mathrm{C}$, and reported a cumulative drug release of $33 \%$ and $37 \%$ of gentamicin from gentamicin 
loaded PHBV. Daniela et al. (2015) observed the release of BSA from loaded PHBV and reported that $47 \%$ drug release followed by $60 \%$ and $63 \%$ in next 21 days. Gursel et al. (2008) observed the controlled release of gentamicin at $22 \%$ for a period of 2 months. Rapid early phase followed by slower and prolonged phase was observed by them. Li and Chang (2005) also observed the controlled release of gentamicin at $70 \%$ during first three days in a period of $12 \mathrm{wks}$ in SBF and PBS.

Poly (3-hydroxybutyrate-co-3-hydroxyvalerate) microspheres were developed with a mean particle size $4 \pm 2 \mu \mathrm{m}$ loaded with daidzein using oil-in-water single emulsion solvent

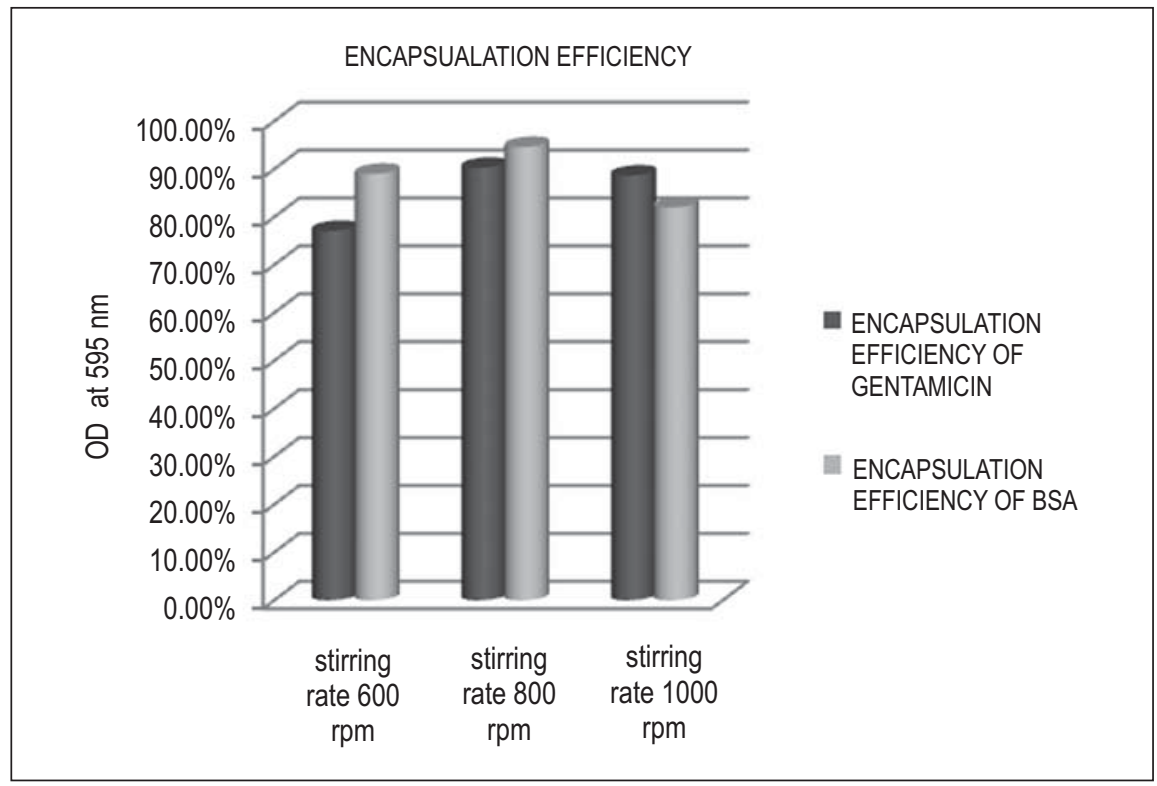

Fig. 6 : Encapsulation efficiency of PHB microsphere encapsulated with gentamicin and BSA at different stirring rate

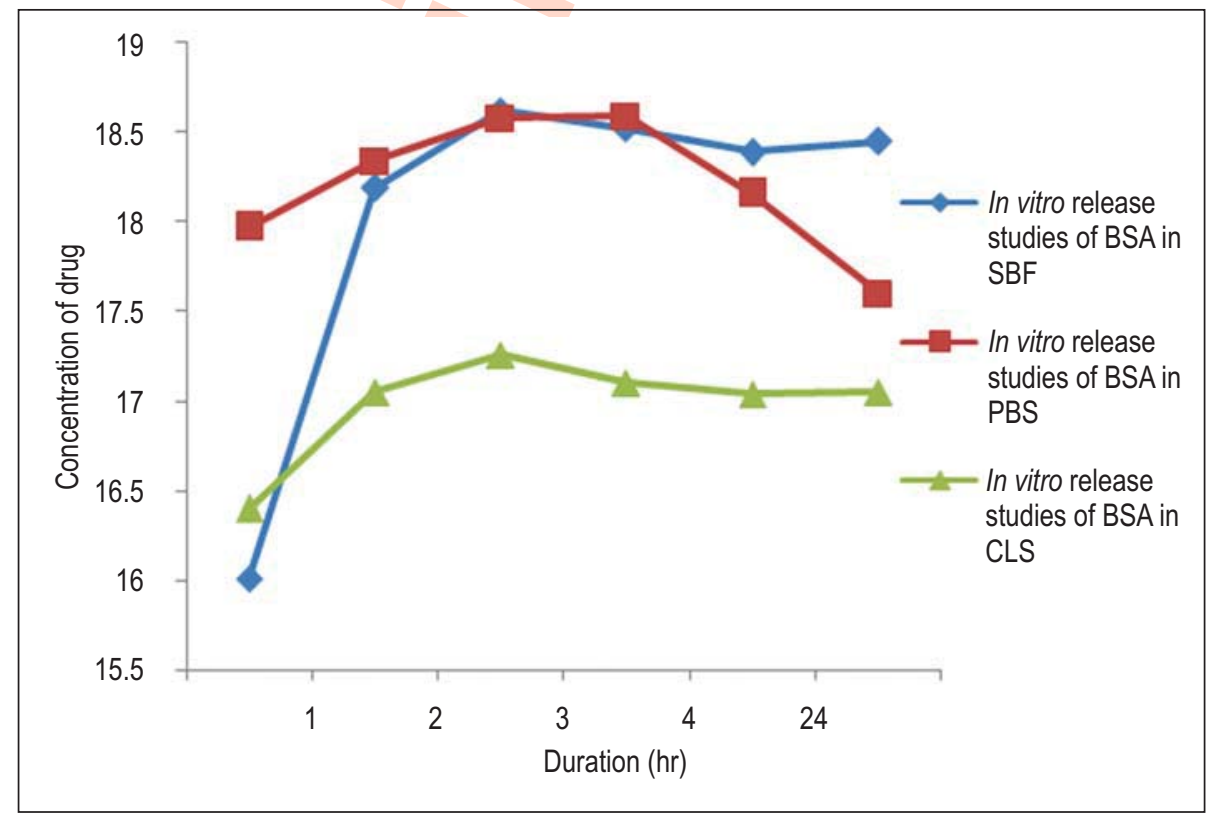

Fig. 7 : In vitro release of BSA from PHB microspheres 
evaporation method and applied to the surface of bio active scaffolds by dip coating technique and also reported that the daidzein release from the microsphere loaded scaffolds lasted for almost one month. (Macías-Andrés et al., 2017)

In the present study, the percentage viability of cells in 3T3L1 cell line at $1 \mathrm{mg}, 10 \mathrm{mg}$ and $100 \mathrm{mg}$ of concentration of PHB microspheres was found to be $97 \%, 87 \%$ and $53 \%$, respectively. At same concentration, the percentage viability of PHB microsphere encapsulated with BSA and gentamicin was found to be $98 \%, 91 \%, 32 \%$ and $95 \%, 53 \%, 47 \%$ respectively (Fig.9). On increasing the concentration of PHB microspheres, decrease in cell viability was observed. Similarly, Shishatskaya et al. (2008) used 3T3 mouse fibroblast cell lines to evaluate the biocompatibility (in vitro cytotoxicity) of PHB microsphere. PHB microspheres showed $99.8 \%$ cell viability and did not produce any toxic effects in $3 \mathrm{~T} 3$ cell line. As the concentration increased, it became toxic to cell lines. In the present study, $98 \%$ cell viability was observed for PHB microspheres at lowest concentration.

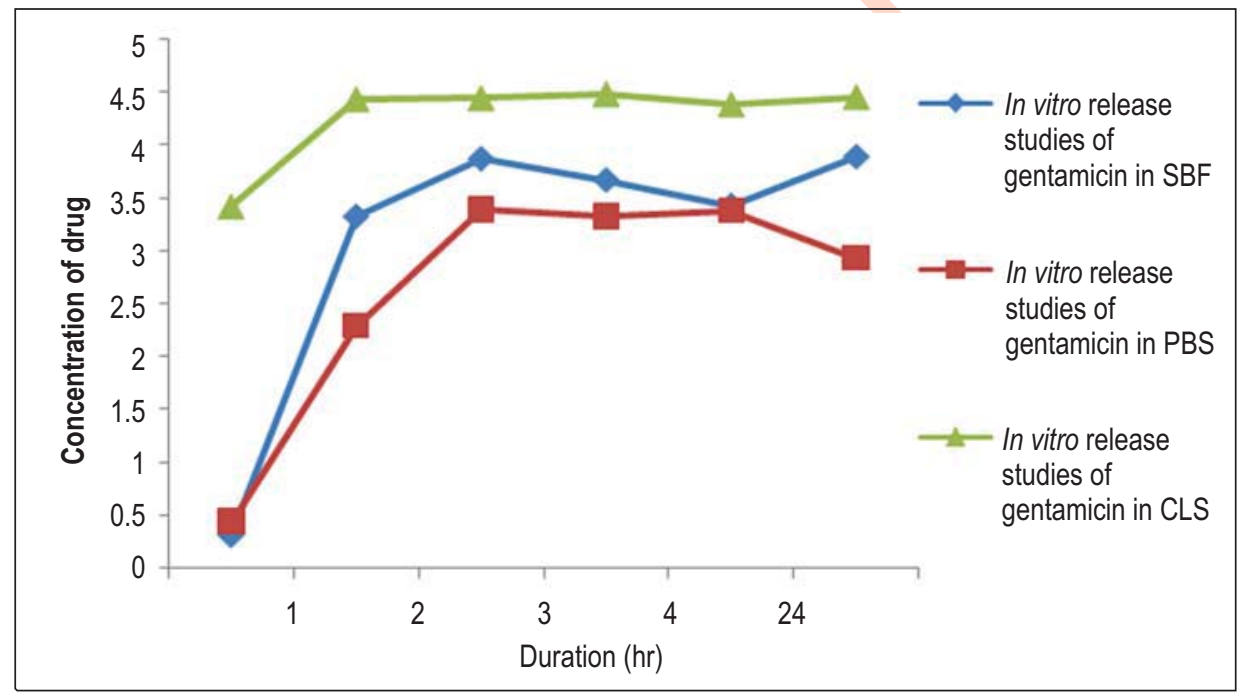

Fig. 8 : In vitro release of Gentamicin from PHB microspheres

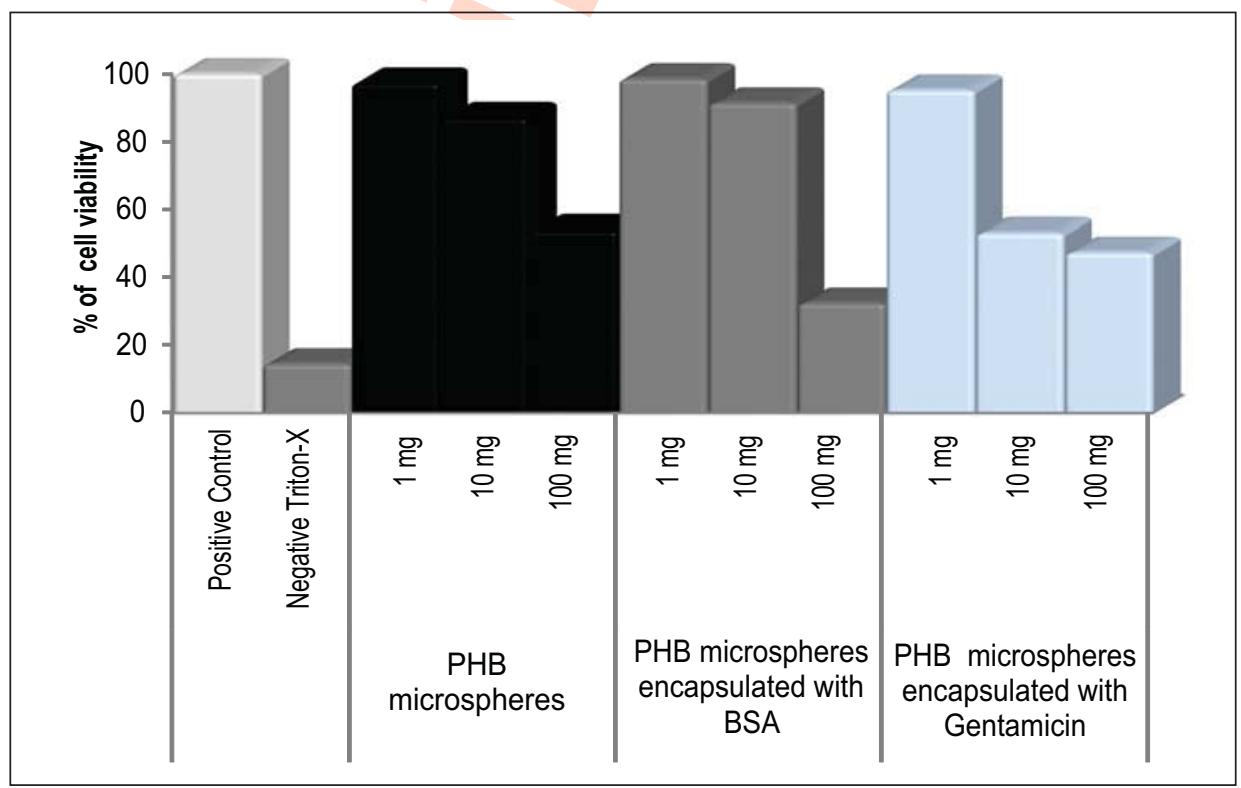

Fig. 9 : In vitro cytotoxicity assay of PHB microsphere encapsulated with BSA and gentamicin in 3T3L1 cell line 
It can be concluded that the microspheres prepared by using PHB produced by Bacillus sp. by solvent evaporation method using dichloromethane and PVA at $1 \%$ concentration at 800rpm showed better encapsulation efficiency when compared to others. Also, the in vitro drug release studies with PHB microspheres encapsulated with BSA and gentamicin showed initial burst release, followed by controlled drug release. Microspheres prepared from PHB produced by Bacillus sp. can be used for encapsulation of antibiotic for controlled drug release.

\section{Acknowledgments}

Authors thank and acknowledge the help rendered by Department of Biotechnology, School of Bioengineering, SRM University for FTIR analysis.

\section{References}

Aggarwal, S., A. Goel and S. Singla: A review on drug delivery: Special emphasis given on biodegradable polymers. Int.J. Sci., 2, 1-15 (2012).

Atul, N.W.: Biosynthesis of polyhydroxybutyrate (PHB): An ecofriendly biopolymer with therapeutic application. Int. J. Inform. Futur. Res., 4, 1068-1080 (2014).

Daniela, P.P., M. H. Amaral, R.L. Reis, A.P. Marques and V.M. Correlo: Development of injectable PHBV microparticles-CG hydrogen hybrid system for regenerative medicine. Int. J. Pharm., 478, 398408 (2015).

Emily, N. and U. Halden Rolf: Plastics and environmental health : The road ahead. Rev. Environ. Hlth., 28, 1-8 (2013).

Francis, L., D. Meng, J. Knowles, T. Keshavarz, A.R. Boccaccini and I. Roy: Controlled delivery of gentamicin using poly (3hydroxybutyrate) microspheres. Int. J. Mol. Sci., 12, 4294-4314 (2011).

Giovana, B.C., A.T. Macedo, J. P. Crenca, V.E. Silva, E. M. Pereira, M. Zetola and B.R. Pezzini: Microspheres prepared with biodegradable PHBV and PLA polymers as prolonged-release system for ibuprofen : In vitro drug release and in vivo evaluation. Braz. J. Pharm. Sci., 48, 773-780 (2012).

Grillo, R., A.E. Pereira, N.F. de Melo, R. M. Porto, L.O. Feitosa, P.S. Tonello, N. L. Dias Filho, A.H. Rosa, R. Lima and L.F. Fraceto: Controlled release system for ametryn using polymer microspheres : Preparation, characterization and release kinetics in water. J. Hazard. Mater., 186, 1645-1651 (2011).
Gursel, I., F. Yagmurulu, F. Korkusuz and V. Hasirci : The In vitro antibiotic release of PHBV rods. J. Microencapsulation: Micro and Nanocarriers, 19, 153-164 (2008).

Ismail, A.F., A.M. Awang and M. Farahidah : High initial burst release of gentamicin formulated as PLGA microspheres implant for treating orthopaedic infection. Int. J. Pharm. Pharm. Sci., 4, 685-691 (2012).

Joshi, J.R. and R. P. Patel: Role of biodegradable polymers in drug delivery. Int. J. Curr. Pharmaceut. Res., 4, 74-81 (2012).

Kokubo,T., H. Kushitani, S. Sakka, T. Kitsugi and T. Yamamuro: Solutions able to reproduce in vivo surface-structure changes in bioactive glass-ceramic. J. Biomed. Mater.Res., 24, 721-734 (1990).

$\mathrm{Li}, \mathrm{H}$. and J. Chang, : Preparation, characterization and in vitro release of gentamicin from PHBV/wollastonite composite microspheres. J. Controll. Rele., 107, 463-473 (2005).

Macias-Andrés, V.I., W. Li, E.A. Aguilar-Reyes, Y. Ding, J.A. Roether, L. Harhaus, C.A. León-Patina and A.R. Boccaccini: Preparation and characterization of $45 S 5$ bioactive glass-based scaffolds loaded with PHBV microspheres with daidzein release function. $J$. Biomed. Mater. Res. Part A., 105(A), 1765-1774 (2017).

Mao, S., C. Guo, Y. Shi and LC. Li: Recent advances in polymeric microspheres for parenteral drug delivery—part 2. Expert Opinion on Drug Delivery., 9, 1209-1223 (2012).

Muralidharan, R. and K.V. Radha: A kinetic study of polyhydroxybutyrate production on nitrogen limited medium using Bacillus subtilis MTCC 9763 through a two stage cultivation strategy. J. Environ. Biol., 36, 537-542 (2015).

Rossi, S., A.O. Azghani and A. Omri: Antimicrobial efficacy of a new antibiotic-loaded poly (hydroxybutyric-co-hydroxyvaleric acid) controlled release system. J. Antimicro. Chemother., 54, 1013$1018(2004)$

Shishatskaya, E. I., O.N. Voinova, A.V. Goreva, O. A. Mogilnaya and T.G. Volova: Biocompatibility of polyhydroxybutyrate microspheres: In vitro and in vivo evaluation. J. Mater. Sci.: Mater. Med., 19, 2493-2502 (2008).

Siracusa, V., P. Rocculi, S. Roamin and M.D. Rosa: Biodegradable polymers for food packaging : A review. Trends in Food Sci. Techno., 19, 634-643 (2008).

Siraj, S., P. Sudhakar, B. Mallikarjuna, K. Chowdoji Rao and M.C.S. Subha : Biodegradable PHBV/PEO blend microspheres for controlled release of Bosentan monohydrate: Preparation, characterization and in vitro release studies. Int. J. Pharm. Sci. Rev. Res., 25, 103-109 (2014).

Yang, Y.Y., T.S. Chung and N.P. Ng: Morphology, drug distribution and in vitro release profiles of biodegradable polymeric microspheres containing protein fabricated by double-emulsion solvent extraction/ evaporation method. Biomaterials, 22, 231-241 (2001). 